\title{
ROLE OF VITAMIN D IN ETIOPATHOGENESIS AND METABOLIC ABNORMALITIES SEEN IN POLYCYSTIC OVARIAN SYNDROME
}

\author{
SURANKITA SUKUL ${ }^{1}$, JYOTIRMAYEE BAHINIPATI ${ }^{2 *}$, ASHOK KUMAR DAS ${ }^{2}$ \\ ${ }^{1}$ Department of Biochemistry, Burdwan Medical College, West Bengal, India. ${ }^{2}$ Department of Biochemistry, Kalinga Institute of Medical \\ Sciences, Bhubaneswar, Odisha, India. Email: jyotirmayee.bahinipati@kims.ac.in
}

Received: 01 April 2019, Revised and Accepted: 25 July 2019

\begin{abstract}
Objective: Polycystic ovarian syndrome (PCOS) is a common cause of ovarian dysfunction in women in reproductive age group. It is now the leading cause of infertility among premenopausal women. PCOS women usually suffer from metabolic disturbances and insulin resistance (IR). Vitamin D has shown a significant role in glucose and insulin metabolism. Correlation studies have been done to examine the role of vitamin D in PCOS. However, still, Vitamin D status in PCOS remains varied.
\end{abstract}

This study is an attempt to find out the association of Vitamin D with etiopathogenesis and metabolic risk factors seen in PCOS.

Methods: Hundred subjects (50 PCOS and 50 age-matched normal control) were recruited for the study. Difference in biochemical parameters in PCOS women and normal group was measured, and association of Vitamin D with etiological and biochemical parameters in PCOS was seen.

Results: There was a significant $(\mathrm{p}<0.001)$ increase in body mass index, serum insulin, fasting blood sugar (FBS), serum cholesterol, triglyceride, and low-density lipoprotein in PCOS. IR was observed in PCOS cases (homeostatic model assessment for $\beta$-cell function and IR $=6.40 \pm 1.96$ ) compared to the control group (2.43 \pm 0.53$)$. Serum $25(\mathrm{OH})$ Vitamin D3 was significantly decreased in PCOS $(9.04 \pm 2.60 \mathrm{ng} / \mathrm{ml}) \mathrm{compared}$ to control group $(20.06 \pm 3.28 \mathrm{ng} / \mathrm{ml})$. Negative correlation of serum Vitamin D was found with FBS, serum insulin, IR, HI, and serum testosterone. Vitamin D with metabolic parameters also showed a statistically significant negative correlation.

Conclusion: Vitamin D deficiency may be a common comorbid manifestation of PCOS. Hence, Vitamin D supplementation may decrease the potential risk of morbidity and mortality associated with PCOS. However, further studies are needed which should include assessment of Vitamin D in women at various stages of PCOS to enhance the temporal order of Vitamin D deficiency in relation to PCOS.

Keywords: Polycystic ovarian syndrome, Vitamin D, Homeostatic model assessment for $\beta$-cell function and insulin resistance, Hirsutism index.

(C) 2019 The Authors. Published by Innovare Academic Sciences Pvt Ltd. This is an open access article under the CC BY license (http://creativecommons. org/licenses/by/4. 0/) DOI: http://dx.doi.org/10.22159/ajpcr.2019.v12i9.33310

\section{INTRODUCTION}

Polycystic ovarian syndrome (PCOS) is a multifactorial, genetically complex endocrine disorder of uncertain etiology and complicated pathophysiology. It is the most common hormonal and metabolic disorder among women in fertile age group [1]. It is characterized by menstrual disturbances, clinical and biochemical manifestations of hyperandrogenism, and polycystic ovaries [2]. It is now the leading cause of anovulatory infertility among premenopausal women. PCOS women are also susceptible to the risks of obesity, insulin resistance (IR), type 2 diabetes mellitus (DM), premature arteriosclerosis, and endometrial cancer [3].

Depending on the criteria for PCOS diagnosis, different prevalence rates have been reported. According to the criteria of the National Institute of Health, $4-10 \%$ of women in their reproductive age suffer from PCOS, whereas in accordance with the Rotterdam criteria, occurrence ranges from $3 \%$ to $26 \%$ in adolescent girls [3]. India has seen a rise in the incidence of PCOS in recent years, which is the direct precursor of anovulatory infertility and diabetes in later life [4]. Although no definitive cause of PCOS had been found, environmental and genetic factors have been linked with the etiopathogenesis of this disorder. Studies have reported the association of Vitamin D receptor (VDR) and Vitamin D level-related variants with metabolic and endocrine parameters in women with PCOS. Vitamin D is thought to influence the development of PCOS through gene transcription, hormonal modulation influencing insulin metabolism and fertility regulation [5]. Many studies have been conducted to understand the mechanism of metabolic disturbances in PCOS [6,7]. A link between Vitamin D and IR was also tried to find out as poor Vitamin D status was associated with IR in Type 2 DM [5]. However, still, the causal relationship between the Vitamin D status and IR remains unclear and varied.

The presence of diverse clinical manifestations of PCOS and its association with long-term health risks has drawn attention to the basic importance of the management of PCOS as a chronic disease. PCOS and its associated complications need to be handled as not a single entity but as a comprehensive clinical system.

This study is an attempt to find out the association of Vitamin D in PCOS patients within the reproductive age group, find out its relationship with etiology and pathogenesis of PCOS and role of Vitamin D and its correlation with the metabolic risk factors such as serum cholesterol, triglyceride (TG), low-density lipoprotein (LDL), high-density lipoprotein (HDL), and body mass index (BMI).

\section{METHODS}

\section{Study design}

This study was done in the department of biochemistry in collaboration with the department of obstetrics and gynecology for a period of 2 years (December 2015-December 2017). The study was started with prior approval from the institutional ethical committee. Written informed consent was taken from the study group after explaining the study objectives. 
A total of 100 subjects of the age group of 15-45 years attending gynecology and obstetrics clinic were recruited for this study. Patients fulfilling at least two criteria of PCOS according to Rotterdam's criteria were selected as cases [8]. Age-matched normal controls were taken for the study purpose. Patients with other hormonal disorders having similar clinical features like adult-onset congenital adrenal hyperplasia, hyperprolactinemia, ovarian androgen producing adenomas, hyperthecosis, Cushing's syndrome, liver and renal disorders, DM, and medications containing sex steroids within 3 months were excluded from the study.

\section{Data collection}

The present and past histories were collected and anthropometric measurements were recorded for each subject. Hirsutism was measured by Ferriman-Gallwey (FG) score. A score of $>8$ was considered as hirsute [8].

\section{Biochemical analysis}

About $5 \mathrm{ml}$ of the venous blood sample was collected under aseptic conditions. Biochemical parameters such as fasting blood sugar (FBS), serum calcium, lipid profile (cholesterol, TG, HDL, LDL, and very LDL [VLDL]), serum insulin, testosterone, follicle-stimulating hormone (FSH), luteinizing hormone (LH), and Vitamin D were evaluated. All biochemical parameters were assayed by Cobas Integra 400 plus (Roche) autoanalyzer. Vitamin D was analyzed by the electrochemiluminescent method by Cobas e411.

IR was estimated by the Homeostatic model assessment for $\beta$ cell function and Insulin Resistance (HOMA-IR) according to the formula: (Fasting insulin $[\mathrm{mU} / \mathrm{L}] \times$ Fasting glucose $[\mathrm{mM} / \mathrm{L}] / 22.5$ ) [7]

Insulin sensitivity was calculated by quantitative insulin sensitivity check index according to the formula: $1 / \log$ (fasting insulin [mU/L]) + $\log$ (fasting glucose [mg/dL]) [7].

\section{Statistical analysis}

All results were expressed in mean $\pm \mathrm{SD}$. The unpaired t-test was done to see for the difference in parameters in the study group. Correlation between Vitamin D and etiological and metabolic parameters was found out by Pearson's correlation coefficient. All the statistical tests were performed by STATA 15.1 version. $\mathrm{p}<0.05$ was considered as statistically significant.

\section{RESULTS}

Table 1 shows the biochemical parameters in the study group. There is a significant increase in BMI, serum insulin, FBS, serum cholesterol,
TG, and LDL in PCOS cases. Statistically significant IR was seen in PCOS cases compared to the control group.

Serum calcium and Vitamin D were significantly decreased in PCOS compared to a normal control group.

Tables 2 and 3 show a statistically significant $(p<0.001)$ positive correlation between serum testosterone with FG score and HOMA-IR with serum testosterone and FG score in PCOS cases.

The correlation of Vitamin D with etiological parameters in PCOS cases is depicted in Table 4 . There is a statistically significant negative correlation $(\mathrm{p}<0.001)$ of serum Vitamin D with FBS, serum insulin, IR, $\mathrm{HI}$, and serum testosterone, whereas there is no significant correlation of Vitamin D which was seen with LH/FSH ratio.

A statistically significant negative correlation was seen between Vitamin D and metabolic parameters (BMI, cholesterol, TG, and LDL) in PCOS cases (Table 5).

\section{DISCUSSION}

PCOS is a heterogeneous androgen excess disorder with different degrees of reproductive and metabolic dysfunctions.

The present study consisted of 100 cases which comprised 50 PCOS and 50 normal healthy controls. Maximum individuals were under the age group of $15-25$ years ( $64 \%$ of PCOS cases and $74 \%$ in control)

BMI of PCOS cases was $\left(25.63 \pm 4.04 \mathrm{~kg} / \mathrm{m}^{2}\right)$ significantly higher than the control group $\left(21.40 \pm 1.75 \mathrm{~kg} / \mathrm{m}^{2}\right)$. In the study done by Beydoun et al., BMI of $30.7 \pm 8.9 \mathrm{~kg} / \mathrm{m}^{2}$ was seen in PCOS cases and suggested that BMI is a prognostic factor for various fertility treatments and hence suggested that obesity adversely affects the outcome of ovulation induction [9]. Lim et al. and Nehra et al. have suggested in their study that there is a greater prevalence of overweight and obesity in women with PCOS compared to normal control. They also found out an independent association of PCOS status with BMI which may be partially attributable to higher energy intake and sedentary lifestyle $[10,11]$. FBS in our study was higher in PCOS cases $(111.74 \pm 15.06 \mathrm{mg} / \mathrm{dl})$ compared to normal control women $(89.66 \pm 14.38 \mathrm{mg} / \mathrm{dl})$. Our finding was in consistence with many studies $[12,13]$. Fasting serum insulin in PCOS was found to be $23.14 \pm 4.58 \mu \mathrm{U} / \mathrm{ml}$ which was raised statistically significantly $(\mathrm{p}<0.001)$ compared to healthy controls $(11.25 \pm 1.41 \mu \mathrm{U} / \mathrm{ml})$. Similar results were also found by Selimoglu et al. with mean basal serum

Table 1: Biochemical parameters in PCOS cases and normal control

\begin{tabular}{llll}
\hline Parameters & PCOS cases (n=50) (Mean \pm SD) & Normal control (n=50) (Mean \pm SD) & p value \\
\hline Hemoglobin (g \%) & $11.82 \pm 1.72$ & $11.92 \pm 1.73$ & 0.77 \\
SBP (mmHg) & $122.4 \pm 12.70$ & $120.3 \pm 12.22$ & 0.40 \\
DBP (mmHg) & $80.1 \pm 7.59$ & $78 \pm 8.86$ & 0.20 \\
BMI $\left(\mathrm{kg} / \mathrm{m}^{2}\right.$ ) & $25.63 \pm 4.04$ & $21.40 \pm 1.75$ & $<0.001^{* *}$ \\
Serum insulin & $23.14 \pm 4.58$ & $11.25 \pm 1.41$ & $<0.001^{* *}$ \\
FBS $(\mathrm{mg} / \mathrm{dl})$ & $111.74 \pm 15.06$ & $89.66 \pm 14.38$ & $<0.001^{* *}$ \\
HOMA-IR & $6.40 \pm 1.96$ & $2.43 \pm 0.53$ & $<0.001^{* *}$ \\
QUICKI & $0.29 \pm 0.01$ & $0.33 \pm 0.01$ & $<0.001^{* *}$ \\
Serum testosterone & $84.6 \pm 12.48$ & $26.38 \pm 4.78$ & $<0.001^{* *}$ \\
HI & $13.17 \pm 4.69$ & $1.16 \pm 0.86$ & $<0.001^{* *}$ \\
LH/FSH & $2.98 \pm 0.14$ & $1.52 \pm 0.12$ & $<0.001^{* *}$ \\
Serum cholesterol $(\mathrm{mg} / \mathrm{dl})$ & $188.56 \pm 2.84$ & $144.2 \pm 19.44$ & $<0.001^{* *}$ \\
Serum triglyceride (mg/dl) & $164.16 \pm 17.20$ & $107.48 \pm 8.94$ & $<0.001^{* *}$ \\
HDL $(\mathrm{mg} / \mathrm{dl})$ & $47.08 \pm 4.88$ & $36.14 \pm 5.11$ & $<0.001^{* *}$ \\
LDL $(\mathrm{mg} / \mathrm{dl})$ & $108.25 \pm 15.80$ & $85.35 \pm 14.40$ & $<0.001^{* *}$ \\
VLDL (mg/dl) & $32.84 \pm 3.44$ & $22.69 \pm 5.90$ & $<0.001^{* *}$ \\
Vitamin D (ng/ml) & $9.04 \pm 2.60$ & $20.06 \pm 3.28$ & $<0.001^{* *}$ \\
Serum calcium (mg/dl) & $6.84 \pm 1.14$ & $9.14 \pm 0.88$ & $<0.001^{* *}$ \\
\hline
\end{tabular}

**Statistically significant. SBP: Systolic blood pressure, DBP: Diastolic blood pressure, FBS: Fasting blood sugar, HOMA-IR: Homeostatic model assessment for $\beta$-cell function and insulin resistance, QUICKI: Quantitative insulin sensitivity check index, HI: Hirsutism index, LH: Luteinizing hormone, FSH: Follicle-stimulating hormone, HDL: High-density lipoprotein, LDL: Low-density lipoprotein, VLDL: Very low-density lipoprotein 
Table 2: Correlation between serum testosterone and FG score

\begin{tabular}{lll}
\hline Parameters & Testosterone & \\
\cline { 2 - 3 } & r value & p value \\
\hline FG score & 0.734 & $<0.001^{* *}$ \\
\hline
\end{tabular}

**Statistically significant

Table 3: Correlation between HOMA-IR with serum testosterone and FG score

\begin{tabular}{lll}
\hline Parameters & HOMA-IR & \\
\cline { 2 - 3 } & r value & p value \\
\hline Testosterone & 0.66 & $<0.001^{* *}$ \\
FG score & 0.721 & $<0.001^{* *}$ \\
\hline
\end{tabular}

**Statistically significant. HOMA-IR: Homeostatic model assessment for $\beta$-cell function and insulin resistance

Table 4: Correlation between Vitamin D and etiological parameters in PCOS cases

\begin{tabular}{lll}
\hline Parameters & Vitamin D & \\
\cline { 2 - 3 } & r value & p value \\
\hline FBS & -0.66 & $<0.001^{* *}$ \\
Serum insulin & -0.86 & $<0.001^{* *}$ \\
HOMA-IR & -0.83 & $<0.001^{* *}$ \\
QUICKI & 0.87 & $<0.001^{* *}$ \\
HI & -0.88 & $<0.001^{* *}$ \\
Testosterone & -0.91 & $<0.001^{* *}$ \\
LH/FSH & -0.12 & NS $(0.40)$ \\
\hline
\end{tabular}

**Statistically significant. NS: Non-significant, FBS: Fasting blood sugar, HOMA-IR: Homeostatic model assessment for $\beta$ cell function and insulin resistance, QUICKI: Quantitative insulin sensitivity check index, HI: Hirsutism index, LH: Luteinizing hormone, FSH: Follicle-stimulating hormone, PCOS: Polycystic ovarian syndrome

Table 5: Correlation between Vitamin D and metabolic parameters in PCOS cases

\begin{tabular}{lll}
\hline Parameters & Vitamin D & \\
\cline { 2 - 3 } & r value & p value \\
\hline BMI & -0.45 & $<0.001^{* *}$ \\
Serum cholesterol & -0.74 & $<0.001^{* *}$ \\
Serum triglyceride & -0.79 & $<0.001^{* *}$ \\
LDL & -0.52 & $<0.001^{* *}$ \\
\hline
\end{tabular}

**Statistically significant. BMI: Body mass index, LDL: Low-density lipoprotein, PCOS: Polycystic ovarian syndrome

insulin levels in PCOS subjects $(14.85 \pm 9.36 \mu \mathrm{U} / \mathrm{ml})$ [14]. Similarly, hyperinsulinemia was also suggested by Wanderley et al. suggesting PCOS leads to many metabolic and reproductive morbidities [15].

HOMA-IR predicts the homeostatic concentrations that arise from varying degrees of IR and beta-cell functions. It has been extensively used to quantify IR and beta-cell function [8]. In our study, mean HOMAIR in PCOS cases was $6.40 \pm 1.96$ and $2.43 \pm 0.53$ in controls. About $92 \%$ of PCOS subjects in our study were having IR. There was a significant correlation between IR in obese PCOS women $(\mathrm{p}<0.001)$. In a systemic review and meta-analysis done by Gandervani et al., PCOS status intensifies the adverse effects of obesity on IR [16].

IR in PCOS is due to post-binding defect in signal transduction. There is a constitutive increase in serine phosphorylation of the insulin receptor and insulin receptor substrate- 1 that inhibits metabolic signaling. Many women with PCOS also exhibit $\beta$-cell dysfunction, rendering insulin response to a glucose load insufficient for the degree of IR in PCOS [17].
Hence, PCOS women should be advised for lifestyle modifications and should be rescreened at least once every 2 years.

Hyperandrogenism is a defining characteristic of PCOS. The most common sign clinically of hyperandrogenism in PCOS in women is hirsutism. In our study, $72 \%$ of PCOS women had FG score $(>8)$ and a severe degree of hirsutism $(>16)$ was found in $28 \%$ of PCOS women. The biochemical estimation of hyperandrogenism is done by the analysis of serum testosterone. Our study showed serum testosterone in PCOS $(84.60 \pm 12.48 \mathrm{ng} / \mathrm{dl})$ which was significantly higher compared to controls $(26.38 \pm 4.78 \mathrm{ng} / \mathrm{dl})$. A similar study done by Jayagopal et al. found higher testosterone in PCOS $(3.9 \pm 0.8 \mathrm{nmol} / \mathrm{l}$ vs. $3.2 \pm 1.3 \mathrm{nmol} / \mathrm{l}$ in normal control, $\mathrm{p}<0.001$ ) [18]. Similar results were also found by Gonzalez F $(83.1 \pm 7.0$ vs. $36.9 \pm 3.7 \mathrm{ng} / \mathrm{ml}$ in PCOS and normal control group, respectively) [19]

There was a statistically significant $(\mathrm{p}<0.001)$ correlation between FG score and testosterone in PCOS, suggesting serum testosterone which is a biochemical marker of hyperandrogenism in females, correlates well with the clinical finding that is hirsutism. A significant correlation was also found between serum testosterone and FG score with HOMA-IR and fasting serum insulin in PCOS, suggesting the relationship of serum testosterone and hyperandrogenism with IR. Hence, increase in insulin levels due to IR is associated with hirsutism in PCOS. The actual role of insulin in the development and maintenance of hyperandrogenism is unclear. In vivo and in vitro studies have stated that insulin acts at multiple sites, leading to increased androgen excess. A common molecular pathway has been hypothesized, i.e., hyperactivity of a single serine kinase for two major features of PCOS - IR and hyperandrogenism. Insulin seems to stimulate both ovarian androgenesis and adrenal androgen synthesis. Thus, a reduction of serum insulin may decrease some androgen-related symptomatology in PCOS women [20].

LH/FSH ratio was found to increase in our study with the sample collected between the day $2^{\text {nd }}$ and $5^{\text {th }}$ of menstrual cycle in PCOS $(2.98 \pm 0.14)$ and control women $(1.52 \pm 0.12)$; similar elevated ratio $(>2)$ in PCOS women with normal and hyperinsulinemia was seen in studies done by Banaszewska et al. [21] and Sharquie et al. [22]. However, a contradictory finding was seen in the study done by Cho et al., LH/FSH ratio did not differ significantly between PCOS and non-affected group. According to them, it was suggested that not every woman with PCOS has an abnormal LH/FSH ratio nor they all possess the hormonal and biochemical changes suggested to the disease [23].

The classic form of PCOS has been associated with an inappropriate gonadotropin secretion. Compared to the follicular phase of the normal menstrual cycle, women with PCOS exhibit a disproportionately high LH secretion with relatively constant low FSH secretion. The underlying cause of this pattern of gonadotropin secretion is linked to an accelerated gonadotropin-releasing hormone $(\mathrm{GnRH})$ pulse generator activities and heightened pituitary response to GnRH. Abnormal feedback mechanism by ovarian estrogen results in an increase in $\mathrm{LH}$ release. As a result, there is a derangement in the ratio between FSH and LH levels. It has been postulated that altered inputs to insulin, insulinlike growth factors, and/or sex steroids during a critical developmental phase of puberty lead to this [24].

Serum cholesterol, TG, and LDL were significantly higher, whereas HDL was significantly lower in PCOS cases $(188.17 \pm 20.14$ vs. $144.20 \pm 19.44$, $164.12 \pm 17.22$ vs. $107.48 \pm 8.95,108.25 \pm 15.80$ vs. $85.35 \pm 14.40$, and $47.42 \pm 6.78$ vs. $36.14 \pm 5.11 \mathrm{mg} / \mathrm{dl}$, respectively). Our results were in accordance with many other studies $[25,26]$. Rocha et al. in their study observed that the incidence of dyslipidemia in the PCOS group was twice that of the control group [26]. Dyslipidemia in PCOS may be attributable to hyperinsulinemia and hyperandrogenemia. This causes adipocytes to undergo increased catecholamines induced lipolysis and release of free fatty acids into the circulation. Increased free fatty acids in the liver stimulate secretion of VLDL which ultimately leads to hypertriglyceridemia. Hyperandrogenism has also been linked to hepatic lipase activity which has a role in the catabolism of HDL particles. 
IR has also been associated with a decreased level of HDL cholesterol and increased level of LDL and TG. This scenario increases the risk of metabolic syndrome and coronary artery diseases in PCOS women [6].

25(OH) Vitamin D in our study was significantly lower $(\mathrm{p}<0.001)$ in PCOS cases $(9.04 \pm 2.60 \mathrm{ng} / \mathrm{ml})$ compared to the control group $(20.06 \pm 3.28 \mathrm{ng} / \mathrm{ml})$. Around $84 \%$ of PCOS cases were severely Vitamin D3 deficient $(<12 \mathrm{ng} / \mathrm{ml})$, whereas in the control group, $56 \%$ were showing insufficient Vitamin D3 levels. In the study reported by Wehr Vitamin D levels were compared in a large number of women in PCOS $(n=545)$ to the controls $(n=145)$, and they were found to be $25.7 \mathrm{ng} /$ $\mathrm{ml}$ versus $32.0 \mathrm{ng} / \mathrm{ml}$, respectively [27]. Li et al. found highly variable 25(OH) Vitamin D levels in both PCOS patients and ovulatory controls ranging from less than the detection limit to as high as $128 \mathrm{nmol} / \mathrm{lt}$. Majority $72 \%$ of PCOS subjects were found to be Vitamin D deficient with $44 \%$ severely deficient levels [28]. In a contradictory study done by Kim et al., women with PCOS showed no differences in the levels of 25(OH)D3 or the prevalence of Vitamin D deficiency though majority had mean D3 $<20 \mathrm{ng} / \mathrm{dl}(19.6 \pm 6.6 \mathrm{ng} / \mathrm{ml})$ and $92 \%$ of PCOS subjects shows Vitamin D insufficiency [29]. In interventional studies done by Selimoglu et al. and Rahimi-Ardabili et al. found that mean serum Vitamin D increased from $16.9 \pm 16 \mathrm{ng} / \mathrm{ml}$ to $37.1 \pm 14.6 \mathrm{ng} / \mathrm{ml}$ and $7 \pm 2.80$ to $22.9 \pm 6.14 \mathrm{ng} / \mathrm{ml}$, respectively, after administration of Vitamin D3 orally [14,30].

There was a statistically significant negative correlation between 25(OH) Vitamin D and BMI in PCOS subjects with a lower level of Vitamin D3 in obese compared to overweight subjects.

Inverse associations between Vitamin D3 and BMI are also found in studies done by Wher et al. and Li et al. and with obese PCOS subjects showing less Vitamin D3 compared to non-obese subjects $[27,28]$. Studies in women with PCOS have shown low D3 levels which were significantly determined by the degree of adiposity, so according to our findings and these supporting findings, it seems that Vitamin D deficiency, obesity, and PCOS are related [31].

It has been stated that $>50 \%$ PCOS are obese and increased androgen and IR is the main etiology behind it [32]. Vitamin D is fat soluble and is sequestered in adipose tissue resulting in its low bioavailability. Besides, dietary preferences and Vitamin D metabolism may also differ in obese and non-obese persons [33].

However, one study done by Panidis et al. had reported no differences between D3 levels across a range of BMI [34]

Vitamin D was negatively correlated with fasting serum insulin and HOMA-IR $(\mathrm{p}<0.001)$. A study done by Zhang et al. suggested that a low Vitamin D level is inversely related to HOMA-IR [35]. Studies done by Wher et al. and Kumar et al. showed the association of hypovitaminosis D with IR and obesity in women with PCOS $[27,36]$. VDR complex regulates more than 300 genes including the genes responsible for glucose and lipid metabolism. Vitamin D increases insulin sensitivity by stimulating the expression of insulin receptors and promoting insulin responsiveness for glucose in the target cells. It also improves the betacell function and increases insulin synthesis and release [37].

Our study showed a statistically significant negative correlation between 25(OH)D3 and FG score and serum testosterone. Various observational studies have found a relationship between markers of hyperandrogenism and Vitamin D status. Hirsute women have shown decreased 25(OH)D3 levels compared to BMI matched controls (17 vs. $29 \mathrm{ng} / \mathrm{ml}$ ) [38]. Hahn et al. observed BMI dependent increasing free androgen index and hirsutism score and suggested the relationship between these variables with Vitamin D status [39]. Wehr et al. found hyperandrogenism related to Vitamin D3, but after controlling BMI, Vitamin D status was no longer significant [40].

Effect of Vitamin D on hyperandrogenism mainly mediated by IR. IR increases hyperandrogenism through insulin increasing ovarian androgen production and decreasing sex hormone-binding globulin $[40,41]$.
Vitamin D deficiency may adversely affect the cardiovascular system. VDRs are present in vascular smooth muscle and endothelium. In our study, there was an adverse relationship between Vitamin D, total cholesterol, TG, and LDL, whereas a statistically positive relationship was seen between Vitamin D and HDL in PCOS. Large cohort studies like Li et al. have shown that Vitamin D deficiency is associated with increased risk of cardiovascular disease and cardiovascular mortality [28]. The adverse relationship was also seen by them between Vitamin D, total cholesterol, LDL, and TG in PCOS with a positive correlation between Vitamin D and HDL. Reported improvements were seen in studies done by Zitterman et al. and Wang et al. in serum TG and HDL, without any changes in BMI after administration of Vitamin D, so these studies suggest the role of hypovitaminosis D with metabolic disorder prevalence in PCOS women [41,42].

Serum calcium was significantly lower in PCOS cases $(6.81 \pm 1.14 \mathrm{mg} / \mathrm{dl})$ compared to normal healthy controls $(9.14 \pm 0.88 \mathrm{mg} / \mathrm{dl})$. A significant correlation was found out between 25(OH) Vitamin D3 and serum calcium in PCOS women $(\mathrm{p}<0.001)$. Similar findings were also seen by Firouzabadi et al. and Rashidi et al., calcium and Vitamin D have a direct effect on ovarian and steroid genesis pathway $[43,44]$. Firuzabadi et al. also found that calcium and Vitamin D supplementation can make a positive effect on follicle maturation, menstrual irregularity, and improvement of hyperandrogenism in infertile women with PCOS [43].

\section{Limitations}

Our sample size was quite small; hence, definitive relationship can be concluded in a larger cohort. We did not correlate with the severity of Vitamin D deficiency.

Further studies are needed which should include assessment of Vitamin $D$ in women at various stages of PCOS to enhance the understanding of temporal order of Vitamin D deficiency in relation to PCOS, that is, if Vitamin D deficiency is a determinant of PCOS, metabolic and hormonal dysregulation, or a consequence of metabolic and hormonal dysregulation or both. Although the role of Vitamin D supplementation has been shown in the management of PCOS, this area also requires further investigation. Large randomized control trials are needed for a better understanding of the effect of Vitamin D supplementation in PCOS patients.

\section{CONCLUSION}

Our study suggests the causal association of Vitamin D with IR, leading to overweight or obesity, metabolic abnormalities, dyslipidemia, and hormonal dysfunctions like increase serum testosterone, leading to hirsutism in PCOS. Vitamin D deficiency may be a common comorbid manifestation of PCOS. Hence, Vitamin D supplementation could be a beneficial treatment of PCOS patients to abolish IR and improve obesity, infertility, and hyperandrogenism. This intervention may also decrease the potential risk of mortality and morbidity associated with metabolic syndrome in PCOS.

\section{ACKNOWLEDGMENT}

We gratefully acknowledge the technical team of the Biochemistry Section of Central Laboratory, Kalinga Institute of Medical Sciences and the study subjects who gave their consent for completion of our study.

\section{AUTHORS' CONTRIBUTIONS}

Dr. Surankita Sukul designed and performed the experiments. Dr. Jyotirmayee Bahinipati processed the experimental data, performed the analysis. and drafted the manuscript. Dr. Ashok Kumar Das designed the analysis and supervised the research. All the authors provided critical feedback and helped shape the research, analysis of the data, and final version of the manuscript.

\section{CONFLICTS OF INTEREST}

The authors declare that they have no conflicts of interest. 


\section{REFERENCES}

1. Laiza MS, Aline DS, Claudia BC, Fernanda ST, Roberto PA, Cristina CS. Metformin usage to induce ovulation in women with polycystic ovary syndrome: A meta-analysis. Int J Pharm Pharm Sci 2016;8:86-9.

2. Dennett CC, Simon J. The role of polycystic ovary syndrome in reproductive and metabolic health: Overview and approaches for treatment. Diabetes Spectrum 2015;28:116-20.

3. Barthelmess EK, Naz RK. Polycystic ovary syndrome: Current status and future perspective. Front Biosci (Elite Ed) 2014:6:104-19.

4. Choudhary A, Jain S, Choudhari P. Prevalence and symptomatology of polycystic ovarian syndrome in Indian women: Is there a rising incidence? Int J Reprod Contracept Obstet Gynaecol 2017;6;4971-5.

5. Aghadavod E, Mollaei H, Nouri M, Hamishehkar H. Evaluation of relationship between body mass index with Vitamin D receptor gene expression and vitamin D levels of follicular fluid in overweight patients with polycystic ovary syndrome. Int J Fertil Steril 2017;11:105-11.

6. Rojas J, Chávez M, Olivar L, Rojas M, Morillo J, Mejías J, et al. Polycystic ovary syndrome, insulin resistance, and obesity: Navigating the pathophysiologic labyrinth. Int J Reprod Med 2014;2014:719050.

7. Diamanti-Kandarakis E, Dunaif A. Insulin resistance and the polycystic ovary syndrome revisited: An update on mechanisms and implications. Endocr Rev 2012;33:981-1030.

8. Rotterdam ESHRE/ASRM-Sponsored PCOS consensus workshop group. Revised 2003 consensus on diagnostic criteria and long-term health risks related to polycystic ovary syndrome (PCOS). Hum Reprod 2004;19:41-7.

9. Beydoun HA, Stadtmauer L, Beydoun MA, Russell H, Zhao Y, Oehninger S, et al. Polycystic ovary syndrome, body mass index and outcomes of assisted reproductive technologies. Reprod Biomed Online 2009; 18:856-63.

10. Lim SS, Norman RJ, Davies MJ, Moran LJ. The effect of obesity on polycystic ovary syndrome: A systematic review and meta-analysis. Obes Rev 2013;14:95-109.

11. Nehra J, Kaushal J, Singhal SR, Ghalaut VS. Comparision of myoinositol versus metformin on anthropometric parameters in polycystic ovarian syndrome in women. Int J Pharm Pharm Sci 2017;9:144-8.

12. Bu Z, Kuok K, Meng J, Wang R, Xu B, Zhang H, et al. The relationship between polycystic ovary syndrome, glucose tolerance status and serum preptin level. Reprod Biol Endocrinol 2012;10:10.

13. Bennal A, Sudha K. Effect of PCOS on glucose metabolism. Natl J Physiol Pharm Pharmacol 2013;3:167-70.

14. Selimoglu H, Duran C, Kiyici S, Ersoy C, Guclu M, Ozkaya G, et al. The effect of Vitamin D replacement therapy on insulin resistance and androgen levels in women with polycystic ovary syndrome. J Endocrinol Invest 2010;33:234-8.

15. Wanderley MD, Pereira LC, Santos CB, Cunha VS, Neves MV. Association between insulin resistance and cardiovascular risk factors in polycystic ovary syndrome patients. Rev Bras Ginecol Obstet 2018:40:188-95.

16. Behboudi-Gandevani S, Tehrani FR, Dovom MR, Farahmand M, Khomami MB, Noroozzadeh M, et al. Insulin resistance in obesity and polycystic ovary syndrome: Systematic review and meta-analysis of observational studies. Gynecol Endocrinol 2016;32:343-53.

17. Pauli JM, Raja-Khan N, Wu X, Legro RS. Current perspectives of insulin resistance and polycystic ovary syndrome. Diabet Med 2011;28:1445-54.

18. Jayagopal V, Kilpatrik ES, Jennings PE, Hepburn DA, Atkin SL. Biological variation of testosterone and sex hormone binding globulin as surrogate marker of insulin resistance. J Endocrinol Metab 2003;88:1528-33.

19. González F, Rote NS, Minium J, Kirwan JP. Reactive oxygen speciesinduced oxidative stress in the development of insulin resistance and hyperandrogenism in polycystic ovary syndrome. J Clin Endocrinol Metab 2006;91:336-40.

20. Baptiste CG, Battista MC, Trottier A, Baillargeon JP. Insulin and hyperandrogenism in women with polycystic ovary syndrome. J Steroid Biochem Mol Biol 2010;122:42-52.

21. Banaszewska B, Spaczyński RZ, Pelesz M, Pawelczyk L. Incidence of elevated LH/FSH ratio in polycystic ovary syndrome women with normo and hyperinsulinemia. Rocz Akad Med Bialymst 2003;48:131-4.

22. Sharquie KE, Al-Bayatti AA, Al-Ajeel AI, Al-Bahar AJ, Al-Nuaimy AA.
Free testosterone, luteinizing hormone/follicle stimulating hormone ratio and pelvic sonography in relation to skin manifestations in patients with polycystic ovary syndrome. Saudi Med J 2007;28:1039-43.

23. Cho LW, Jayagopal V, Kilpatrick ES, Holding S, Atkin SL. The LH/ FSH ratio has little use in diagnosing polycystic ovarian syndrome. Ann Clin Biochem 2006;43:217-9.

24. Yarak S, Bagatin E, Hassm MK, Parada MO, Filu ST. Hyperandrogenism and skin, polycystic ovary syndrome and peripheral insulin resistance. An Bras Dermatol 2005;80:395-410.

25. Shoaib OM, Mustafa SM, Nouren IH. Serum lipid profile in polycystic ovarian syndrome in Sudanese women. Int J Med Sci Public Health 2015:4:1605-10.

26. Rocha MP, Marcondes JA, Barcellos CR, Hayashida SA, Curi DD, da Fonseca ÂM, et al. Dyslipidemia in women with polycystic ovary syndrome: Incidence, pattern and predictors. Gynecol Endocrinol 2011;27:814-9

27. Wehr E, Pieber TR, Obermayer-Pietsch B. Effect of vitamin D3 treatment on glucose metabolism and menstrual frequency in polycystic ovary syndrome women: A pilot study. J Endocrinol Invest 2011;34:757-63.

28. Li HW, Brereton RE, Anderson RA, Wallace AM, Ho CK. Vitamin D deficiency is common and associated with metabolic risk factors in patients with polycystic ovary syndrome. Metabolism 2011;60:1475-81.

29. Kim JJ, Choi YM, Chae SJ, Hwang KR, Yoon SH, Kim MJ, et al. Vitamin D deficiency in women with polycystic ovary syndrome. Clin Exp Reprod Med 2014;41:80-5.

30. Rahimi-Ardabili H, Pourghassem Gargari B, Farzadi L. Effects of Vitamin D on cardiovascular disease risk factors in polycystic ovary syndrome women with vitamin D deficiency. J Endocrinol Invest 2013;36:28-32.

31. Lagunova Z, Porojnicu AC, Lindberg F, Hexeberg S, Moan J. The dependency of Vitamin D status on body mass index, gender, age and season. Anticancer Res 2009;29:3713-20.

32. Cebeci F, Onsun N, Mert M. Insulin resistance in women with hirsutism. Arch Med Sci 2012;8:342-6.

33. Vanlint S. Vitamin D and obesity. Nutrients 2013;5:949-56

34. Panidis D, Balaris C, Farmakiotis D, Rousso D, Kourtis A, Balaris V, et al. Serum parathyroid hormone concentrations are increased in women with polycystic ovary syndrome. Clin Chem 2005;51:1691-7.

35. Zhang J, Ye J, Guo G, Lan Z, Li X, Pan Z, et al. Vitamin D status is negatively correlated with insulin resistance in Chinese Type 2 diabetes. Int J Endocrinol 2016;2016:1794894.

36. Kumar A, Barki S, Raghav V, Chaturvedi A, Kumar KV. Correlation of Vitamin D with metabolic parameters in polycystic ovarian syndrome. J Family Med Prim Care 2017;6:115-9.

37. Clemente-Postigo M, Muñoz-Garach A, Serrano M, Garrido-Sánchez L, Bernal-López MR, Fernández-García D, et al. Serum 25-hydroxy Vitamin D and adipose tissue Vitamin D receptor gene expression: Relationship with obesity and Type 2 diabetes. J Clin Endocrinol Metab 2015;100:E591-5.

38. Glintborg D, Andersen M. Management of endocrine disease: Morbidity in polycystic ovary syndrome. Eur J Endocrinol 2017;176:R53-R65

39. Hahn S, Haselhorst U, Tan S, Quadbeck B, Schmidt M, Roesler S, et al. Low serum 25-hydroxy Vitamin D concentrations are associated with insulin resistance and obesity in women with polycystic ovary syndrome. Exp Clin Endocrinol Diabetes 2006;114:577-83.

40. Wehr E, Pilz S, Schweighofer N, Giuliani A, Kopera D, Pieber TR, et al. Association of hypovitaminosis D with metabolic disturbances in polycystic ovary syndrome. Eur J Endocrinol 2009;161:575-82.

41. Zittermann A, Frisch S, Berthold HK, Götting C, Kuhn J, Kleesiek K, et al. Vitamin D supplementation enhances the beneficial effects of weight loss on cardiovascular disease risk markers. Am J Clin Nutr 2009;89:1321-7.

42. Wang TJ, Pencina MJ, Booth SL, Jacques PF, Ingelsson E, Lanier K, et al. Vitamin D deficiency and risk of cardiovascular disease. Circulation 2008;117:503-11.

43. Firouzabadi RD, Aflatoonian A, Modarresi S, Sekhavat L, Taheri SM. Therapeutic effects of calcium and Vitamin D supplementation in women with PCOS. Complement Ther Clin Pract 2012;18:85-8.

44. Rashidi B, Haghollahi F, Shariat M, Zayerii F. The effects of calciumVitamin D and metformin on polycystic ovary syndrome: A pilot study. Taiwan J Obstet Gynecol 2009;48:142-7. 